\title{
Effects of Natural Bioactive Products on the Growth and Ginsenoside Contents of Panax ginseng Cultured in an Aeroponic System
}

\author{
Geum-Soog Kim¹, Seung-Eun Lee ${ }^{1}$, Hyung-Jun Noh ${ }^{1}$, Hyuck Kwon ${ }^{2}$, Sung-Woo Lee ${ }^{1}$, Seung-Yu \\ $\mathrm{Kim}^{1}$, and Yong-Bum Kim ${ }^{{ }^{*}}$ \\ ${ }^{1}$ Department of Herbal Crop Research, National Institute of Horticultural \& Herbal Science, Rural Development Administration, \\ Eumseong 369-873, Korea \\ ${ }^{2}$ International Ginseng \& Herb Research Institute, Geumsan 312-804, Korea
}

This study was conducted to evaluate the effects of natural bioactive products such as Manda enzyme (T1), Yangmyeongwon (T2), effective microorganisms (T3), and Kelpak (T4) on the growth and ginsenoside contents of Panax ginseng cultured in an aeroponic system using a two-layer vertical type of nutrient bath under natural light conditions. The growth of ginseng plants showed specific characteristics according to the positions in which they were cultured due to the difference of light transmittance and temperature in the upper and lower layers during aeroponic culture in a two-layer vertical type of system. The growth of the aerial part of the leaves and stems of ginseng plants cultured in the lower layer $\left(4,000\right.$ to $6,000 \mathrm{~lx}, 23^{\circ} \mathrm{C}$ to $\left.26^{\circ} \mathrm{C}\right)$ of the nutrient bath was observed to be superior to that of the ginseng plants cultured in the upper layer $\left(12,000\right.$ to $15,0001 \mathrm{x}, 25^{\circ} \mathrm{C}$ to $\left.28^{\circ} \mathrm{C}\right)$. The leaf area was significantly larger in the treatment of T2 and T4 $\left(46.70 \mathrm{~cm}^{2}\right)$ than with other treatments. Conversely, the values of the root weight and root diameter were higher in ginseng plants cultured in the upper layer of the nutrient bath. The root weight was significantly heavier in the treatment of T4 $(6.46 \mathrm{~g})$ and T3 $(6.26 \mathrm{~g})$ than with other treatments. The total ginsenoside content in the leaves and roots was highest in the ginseng plants cultured by the treatment of $\mathrm{T} 1$, at $16.20 \%$, while the total ginsenoside content obtained by other treatments decreased in the order of T4, T5 (control), T2, and T3, at 13.21\%, 12.30\%, 14.84\%, and 14.86\%, respectively. The total ginsenoside content of the ginseng leaves was found to be significantly higher in the treatment of $\mathrm{T} 1$ in the lower layer of the nutrient bath, at $15.30 \%$, while the content of the ginseng roots in the treatments of $\mathrm{T} 3$ and $\mathrm{T} 4$, at $1.27 \%$ and $1.23 \%$, respectively, was significantly higher than in other treatments in the upper layer of the nutrient bath.

Keywords: Panax ginseng, Aeroponic system, Ginsenoside, Natural bioactive products

\section{INTRODUCTION}

Ginseng (Panax ginseng Meyer) is a perennial herbaceous plant with a variety of medicinal efficacies that have been recognized and documented throughout the long history of Asian countries such as Korea and China, the major producers and consumers of ginseng. The cultivation and production of American ginseng (P. quinquefolium L.), which is grown in North America (USA and Canada), have increased in recent times, to the extent that it has become another major ginseng species in the world, and consequently leading to an expansion in both interest and consumption. However, these two species of ginseng differ in terms of their components, which have been well documented in various reports, and their efficacies, which have rarely been documented, except in (c) This is an Open Access article distributed under the terms of the Creative Commons Attribution Non-Commercial License (http://creativecommons.org/licenses/by-nc/3.0/) which permits unrestricted non-commercial use, distribution, and reproduction in any medium, provided the original work is properly cited.
Received 30 Aug. 2011, Revised 11 May. 2012, Accepted 29 May. 2012

*Corresponding author

E-mail:kimyb707@korea.kr

Tel: +82-43-871-5544, Fax: +82-2-43-871-5539 
studies on ginseng.

The representative bioactivities of ginseng reported thus far include anti-diabetes [1,2], antioxidant [3,4], antitumor [5-7], neuro-protectiveness, neuro-protectiveness $[8,9]$, liver-function promotion $[10,11]$ and anti-stress $[12,13]$ activities. Although these bioactivities have been frequently applied to remedy human diseases, ginseng products are popularly purchased for use as healthfunctional foods to prevent disease and promote health, as consumers are presently more interested in the prevention of diseases than their remedy. In the case of ginseng, however, only seven kinds of health claims have been approved by the Korea Food and Drug Administration for products currently available on the market, including two health claims, i.e., immunity boosting and fatigue recovery for white ginseng, and five health claims, i.e., immunity boosting, fatigue recovery, antioxidant efficacy, blood flow improvement by inhibiting platelet aggregation, and memory improvement for red ginseng. This is due to a lack of scientific evidence concerning ginseng's efficacy based on clinical testing on human subjects, despite the abundance of experimental reports on ginseng's presumed efficacies. It is predicted that ginseng production will be further expanded to meet the growing market for functional health foods, so continuous attempts will be made to develop a variety of ginseng products for this purpose. Public demand for diverse raw ginseng materials may increase given that the consumption of ginseng products is expanding in line with the increased interest in ginseng as a functional health food.

The hydroponics of ginseng is a representative example of raw ginseng production for use as a functional food material by culturing ginseng seedlings for a short period of 3 to 4 mo under a controlled environment (light, temperature, moisture, and carbon dioxide content) in a high-tech greenhouse, producing not only pesticide-free ginseng roots but also ginseng leaves with a high ginsenoside content. In our previous study [14], the quality of ginseng cultured by a hydroponic system was evaluated by analyzing the ginsenoside content in several parts of a ginseng plant. In addition, wood-cultivated and organically-cultured types of ginseng are further examples of raw ginseng production to meet the current situation in the ginseng market.

Currently, the interest in safe agricultural products of high quality is gradually increasing, leading to an increase in the cultivation acreage by hydroponics for the production of vegetables and flowers in high-tech cultural facilities. This study aims to develop an aeroponic system for the cultivation of ginseng plants for a short period of time (i.e., 4 mo) using ginseng seedlings, which can be utilized as a fresh cut functional vegetable or high-quality food material. Several natural bioactive products popularly used in hydroponic culture systems were examined for their effects on ginseng growth and ginsenoside content in order to provide basic information for the promotion of value-added of ginseng using an aeroponic culture system.

\section{MATERIALS AND METHODS}

\section{Ginseng materials}

Experiments were conducted using the ginseng variety known as Cheonpoong, which is known to be resistant to moisture stress, thus making it suitable for aeroponic and paddy culture. One-year-old ginseng seedling roots weighing 0.7 to $0.8 \mathrm{~g}$ were purchased from a ginseng market and stored in a chamber at low temperature $\left(1^{\circ} \mathrm{C}\right.$ to $2^{\circ} \mathrm{C}$ ) before use. The ginseng seedling roots were transplanted to nutrient baths and cultured in an aeroponic system. After 4 mo of culture, the ginseng plants were pulled out for harvesting. The harvested ginseng plants were washed clean of dust with water and sorted into leaves and roots, which were then dried for $72 \mathrm{~h}$ in a freeze dryer (KR/PVTFD 30A; Ilshin Biobase Co., Yangju, Korea). The freeze-dried ginseng leaves and roots were ground into a powder and used as assay samples in this study.

\section{Chemicals and tools}

Ginsenoside components such as ginsenoside Rb1, Rb2, Rc, Rd, Re, Rf, Rg1, Rg2, and Rh1 were purchased from a chemical company (ChromaDex Inc., Santa Anna, CA, USA). MeOH (Merck \& Co Inc,. Darmstadt, Germany) and other GR-grade solvents were used for the quantitative analysis of ginsenosides by an Agilent 1100 series HPLC system (Agilent Technologies, Palo Alto, CA, USA), using Sep-Pak Plus C18 cartridges (Waters Corp., Milford, MA, USA) for the solid phase extraction (SPE).

\section{Analysis of ginsenosides}

The pretreatment of powdered ginseng samples and the analysis of ginsenosides followed the methods described in a previous study [15]. For each treatment, $2 \mathrm{~g}$ of freeze-dried powder sampled from 30 freeze-dried ginseng plants was used for the extraction of ginsenosides. Each sample was suspended in $40 \mathrm{~mL}$ of $50 \% \mathrm{MeOH}$ in a $50 \mathrm{~mL}$ centrifuge tube with the lid closed, placed in an ultrasonic bath (Powersonic 410; Hwashin Tech., Seoul, 
Korea), extracted by sonication for $15 \mathrm{~min}$, and filtered through Whatman no.1 filter paper (Advantech, Tokyo, Japan). These processes were replicated twice and the resulting filtrates were combined and adjusted to the volume of $100 \mathrm{~mL}$ with $\mathrm{MeOH}$ in a $100 \mathrm{~mL}$-scaled flask, of which $1 \mathrm{~mL}$ was used for pretreatment using SPE before the HPLC analysis. For SPE, a Sep-Pak Plus C18 cartridge was eluted slowly using $3 \mathrm{~mL} \mathrm{MeOH}$ for the lower conditioning, and again using $3 \mathrm{~mL}$ dd $-\mathrm{H}_{2} \mathrm{O}$ for the upper conditioning. One $\mathrm{mL}$ of the extracted solution $(50 \%$ $\mathrm{MeOH}$ extract) was loaded into the cartridge and eluted slowly using $10 \mathrm{~mL}$ dd $-\mathrm{H}_{2} \mathrm{O}$ to remove any sugar soluble materials, then eluted slowly using $2 \mathrm{~mL} \mathrm{MeOH}$ to extract the ginsenoside components. The final eluent was adjusted to an exact volume of $2 \mathrm{~mL}$ and filtered through a membrane filter (with a pore size of $0.45 \mu \mathrm{m}$ ), and then analyzed by HPLC on a YMC-Pack ODS AM column (250×4.6 mm, $5 \mu \mathrm{m}$; YMC Inc., Wilmington, NC, USA) at $43^{\circ} \mathrm{C}$ with a flow rate of $0.8 \mathrm{~mL} / \mathrm{min}$. The gradient elution of the extracts was processed as follows: in $27 \%$ aq. acetonitrile for $10 \mathrm{~min}$; in a gradient concentration of acetonitrile from $27 \%$ to $42 \%$ for $35 \mathrm{~min}$, and from $42 \%$ to $95 \%$ for $2 \mathrm{~min}$; and in $95 \%$ aq. acetonitrile for $15 \mathrm{~min}$. The extracts were analyzed by a UV detector at a wavelength of $203 \mathrm{~nm}$.

\section{Ginseng culture in an aeroponic system and treat- ment with natural bioactive products}

This study was conducted in the greenhouse of the National Institute of Horticultural \& Herbal Science located at Eumseong, Chungbuk Province, Korea for 4 mo from April 20 to August 20, 2009, starting with the transplantation of the ginseng seedlings in a 1-2W type of greenhouse. Cultivation baths were constructed with the two-layer vertical type of benches made with $30 \mathrm{~mm}$ rectangular steel frames. Forty cm high first layer and 160 $\mathrm{cm}$ high second layer with $5.5 \mathrm{~m}$ length for both were welded for fabrication. To provide sufficient intensity of radiation to the ginseng plants by aeroponics, a space of $80 \mathrm{~cm}$ was maintained between the aeroponic systems on the top of the lower layer and bottom of the upper layer.

The aeroponic system was made by joining together polystyrene baths with dimensions of $85 \mathrm{~cm}$ (width) $\times 35$ $\mathrm{cm}$ (height) $\times 4 \mathrm{~cm}$ (thickness), and overlaying the inside of the baths with black and white polyethylene (PE) film for waterproofing. The nutrient supply pipelines were made of PE tubes with an inner diameter of 25 $\mathrm{mm}$, which were laid down on the baths in two rows and equipped with two nozzles with a spraying capacity of $2 \mathrm{~L} / \mathrm{min}$ and a controlled spraying pressure of 1.5 to $2.0 \mathrm{kgf} / \mathrm{cm}^{2}$. The ginseng seedlings were transplanted on April 20, 2009 by inserting them into a polyurethane sponge block $(3 \times 3 \times 3 \mathrm{~cm})$ with holes arranged at a distance of $7 \times 9 \mathrm{~cm}$ from each other on the polystyrene baths. To ensure efficient transplantation, homogeneous naturalization, and timely foliation and plant growth, the plants were transplanted into the lower layer with a favorable environment for transplantation because of the low light intensity, moved to the upper layer after naturalization for 2 to $3 \mathrm{~d}$, and then transplanted into the lower layer again.

Nutrient provision was performed with a nutrient solution (developed exclusively for ginseng by the Rural Development Administration) composed of $\mathrm{NO}_{3} \mathrm{~N}$ 12.0, $\mathrm{NH}_{4} \mathrm{~N}$ 1.0, K 8.0, Ca 4.0, Mg 2.0, $\mathrm{PO}_{4} \mathrm{P} 3.0, \mathrm{SO}_{4} \mathrm{~S} 2.0$ me/L, Fe-EDTA 0.6, Mn 0.5, B 0.5, Cu 0.02, Mo 0.05, and $\mathrm{Zn} 0.05 \mathrm{mg} / \mathrm{L}$, using the aeroponic (water spray) method. The nutrient solution was sprayed for $30 \mathrm{~s}$ every $10 \mathrm{~min}$ in the daytime and for $30 \mathrm{~s}$ every $30 \mathrm{~min}$ in the nighttime using a twin-timer, but for $30 \mathrm{~s}$ every $5 \mathrm{~min}$ in the daytime during the hot summer season.

The natural bioactive products used as treatments in this study were as follows: for treatment 1 (T1), a 10,000 $\mathrm{x}$ diluted solution of Manda enzyme (T1; Manda Fermentation Co., Hiroshima, Japan) made from 50 kinds of plant materials including fruits, citrus, root vegetables, grains, legumes, and seaweeds after microbial culture and fermentation for at least $3 \mathrm{yr}$ for aging was used. Thus, this product is known to contain plant bioactive substances including 18 amino acids like arginine and lysine, nutrients and minerals, which enhance photosynthesis enabling plants to grow stronger. For treatment 2 (T2), an $8,000 \mathrm{x}$ diluted solution of Yangmyeongwon (Dream AGRO, Nonsan, Korea) was used. This product was made from fermenting a mixture of about 70 plant materials such as fruits, vegetables, citrus, root vegetables, and fruit vegetables and 15 microbes such as Mongolian lactobacilli and yeasts at a low temperature in natural environments for more than 3 years. Treatment 3 (T3) was a $3000 \times$ diluted solution of effective microorganisms (EM; EMcenter, Seogwipo, Korea) made from the culture of 80 microbial species such as photosynthetic bacteria, lactobacilli and yeasts, which are known to have remarkable antioxidant and revivifying properties. Treatment 4 (T4) was a 3,000 x diluted solution of Kelpak (T4; Agrichem Ltd., Queensland, Australia), which is a bioactive substance extracted from seaweed (Ecklonia maxima) grown to a giant size, i.e., with a shoot height of 12 to $13 \mathrm{~m}$ and a stem diameter of 7 to $8 \mathrm{~cm}$, and which contains plant growth hormones such as cytokinins, gibberellins, auxins 
and amino acids required for plant growth and cell division, as well as 66 active components. The application of an extremely small amount of this product is effective in promoting plant growth. Untreated control was used as treatment 5 (T5).

The nutrient solution was managed to maintain electric conductivity (EC) at $0.6-0.8 \mathrm{dS} / \mathrm{m}$ and $\mathrm{pH} 6.0 \pm 0.5$; EC of $0.6 \pm 0.1 \mathrm{dS} / \mathrm{m}$ initially, and EC $0.8 \pm 0.1 \mathrm{dS} / \mathrm{m}$ from one month after transplantation to the end. The temperature of the nutrient solution around the rhizosphere was managed at around $21 \pm 1^{\circ} \mathrm{C}$; and the temperature of the aeroponic system was maintained around $23^{\circ} \mathrm{C}$ to $25 \pm 2^{\circ} \mathrm{C}$ in May and June and around $28^{\circ} \mathrm{C}$ to $30 \pm 2^{\circ} \mathrm{C}$ in July and August.

Light intensity on the baths was maintained at 8,000$12,000 \mathrm{~lx}$ using an illumination sensor, which was controlled by a double-layered curtain metalized with $55 \%$ aluminum operating under the horizontal towing method. A tunnel made of white felt $(55 \mathrm{~g})$ was established in the upper portion of the two-layer baths to prevent the leaves from being burnt by the high temperature and strong sunlight at midday.

\section{Statistical analyses}

The values of the growth measurements and ginsenoside content were expressed as mean \pm standard deviations, and conducted in a split plot design. ANOVA were carried out using the SAS (SAS Institute Inc., Cary, NC, USA). Duncan's multiple range test was employed to test for significant differences between the treatments at $p<0.05$.

\section{RESULTS AND DISCUSSION}

\section{Ginseng growth in an aeroponic system influenced by treatment with natural bioactive products}

The ginseng's growth characteristics were examined in 10 plants with 3 replications for each treatment on August 20, 2009 over a harvesting time of $120 \mathrm{~d}$ after transplanting the ginseng seedling roots into the aeroponic system (Table 1). Of the 5 treatments, growths generally improved in T2 and T4. Growth in the aerial part of the ginseng plants was better in those grown in the lower layer baths at a light intensity of 4,000 to 6,000 lx compared with those grown in the upper layer, showing an average shoot height of 20.3 and $18.0 \mathrm{~cm}$ and an average leaf area of 42.7 and $37.8 \mathrm{~cm}^{2}$ for the lower and upper layer plants, respectively. The size of the leaf area increased significantly in T2 $\left(46.70 \mathrm{~cm}^{2}\right), T 4\left(46.70 \mathrm{~cm}^{2}\right)$ and T5 $\left(46.20 \mathrm{~cm}^{2}\right)$ compared to other treatments (Table 1). It was reported in previous studies $[16,17]$ that ginseng growth is significantly influenced by temperature and light intensity; while stem height, leaf length and chlorophyll contents decrease with an increase of light intensity. In our study, too, growth in the aerial part of the ginseng plants was poorer in the upper layer baths with higher light transmissibility than those in the lower layer, which is assumed to be better for ginseng growth in the lower layer of the aeroponic system owing to the relatively lower decrease in the chlorophyll content of the ginseng leaves than in the upper layer illuminated with higher light intensity. Considering the fact that these

Table 1. The growth characteristics of ginseng plants cultured by an aeroponic system using a two-layer vertical type of nutrient bath with different natural bioactive products

\begin{tabular}{|c|c|c|c|c|c|c|c|c|c|c|}
\hline \multicolumn{2}{|c|}{$\begin{array}{l}\text { Natural bioactive } \\
\text { products }^{1 /}\end{array}$} & \multirow{2}{*}{$\begin{array}{c}\begin{array}{c}\text { Plant height } \\
(\mathrm{cm})\end{array} \\
20.30 \mathrm{a}^{2)}\end{array}$} & \multirow{2}{*}{$\begin{array}{c}\begin{array}{c}\text { No. of leaves } \\
\text { (each) }\end{array} \\
2.20 \mathrm{a}\end{array}$} & \multirow{2}{*}{$\begin{array}{c}\begin{array}{c}\text { Leaf length } \\
(\mathrm{cm})\end{array} \\
6.53 \mathrm{ab}\end{array}$} & \multirow{2}{*}{$\begin{array}{c}\begin{array}{c}\text { Leaf area } \\
\left(\mathrm{cm}^{2} / \text { plant }\right)\end{array} \\
35.30 \mathrm{c}\end{array}$} & \multirow{2}{*}{$\begin{array}{c}\begin{array}{c}\text { Stem } \\
\text { length }(\mathrm{cm})\end{array} \\
8.06 \mathrm{a}\end{array}$} & \multirow{2}{*}{$\begin{array}{c}\begin{array}{c}\text { Stem diameter } \\
(\mathrm{mm})\end{array} \\
1.98 \mathrm{ab}\end{array}$} & \multirow{2}{*}{$\begin{array}{c}\begin{array}{c}\text { Root } \\
\text { length }(\mathrm{cm})\end{array} \\
10.30 \mathrm{a}\end{array}$} & \multirow{2}{*}{$\begin{array}{c}\begin{array}{c}\text { Root diameter } \\
(\mathrm{mm})\end{array} \\
9.57 \mathrm{a}\end{array}$} & \multirow{2}{*}{$\begin{array}{c}\text { Root weight } \\
(\mathrm{g})\end{array}$} \\
\hline Lower layer & $\mathrm{T} 1$ & & & & & & & & & \\
\hline & $\mathrm{T} 2$ & $19.50 \mathrm{ab}$ & $2.13 \mathrm{a}$ & $6.84 \mathrm{ab}$ & $46.70 \mathrm{a}$ & $7.49 \mathrm{a}$ & $1.87 \mathrm{~b}$ & $11.90 \mathrm{a}$ & $9.62 \mathrm{a}$ & $6.18 \mathrm{a}$ \\
\hline & $\mathrm{T} 3$ & $19.50 \mathrm{bc}$ & $2.37 \mathrm{a}$ & $7.00 \mathrm{c}$ & $38.70 \mathrm{~b}$ & $8.33 \mathrm{a}$ & $2.04 \mathrm{a}$ & $12.80 \mathrm{a}$ & $9.23 \mathrm{a}$ & $4.71 \mathrm{c}$ \\
\hline & $\mathrm{T} 4$ & $20.70 \mathrm{c}$ & $2.30 \mathrm{a}$ & $7.19 b c$ & $46.70 \mathrm{a}$ & $8.77 \mathrm{a}$ & $1.98 \mathrm{ab}$ & $12.30 \mathrm{a}$ & $9.16 \mathrm{a}$ & $5.33 b$ \\
\hline & T5 & $21.30 \mathrm{bc}$ & $2.13 a$ & $6.90 \mathrm{bc}$ & $46.20 \mathrm{a}$ & $8.19 a$ & $2.02 \mathrm{ab}$ & $10.90 \mathrm{a}$ & $8.19 b$ & $4.47 \mathrm{c}$ \\
\hline & Mean & 20.30 & 2.23 & 6.89 & 42.70 & 8.17 & 1.98 & 11.60 & 9.16 & 5.19 \\
\hline \multirow[t]{6}{*}{ Upper layer } & $\mathrm{T} 1$ & $16.50 \mathrm{~d}$ & $2.27 \mathrm{a}$ & $6.04 \mathrm{~b}$ & $31.50 \mathrm{a}$ & $6.10 \mathrm{a}$ & $2.15 \mathrm{a}$ & $10.30 \mathrm{a}$ & $9.71 \mathrm{ab}$ & $5.23 \mathrm{~d}$ \\
\hline & $\mathrm{T} 2$ & $17.30 \mathrm{~cd}$ & $2.10 \mathrm{a}$ & $6.31 \mathrm{~b}$ & $35.90 \mathrm{ab}$ & $6.20 \mathrm{ab}$ & $2.00 \mathrm{ab}$ & $11.40 \mathrm{a}$ & $9.53 \mathrm{ab}$ & $5.84 \mathrm{bc}$ \\
\hline & $\mathrm{T} 3$ & $18.00 \mathrm{bc}$ & $2.30 \mathrm{a}$ & $6.36 \mathrm{~b}$ & $40.40 \mathrm{~b}$ & $6.90 \mathrm{ab}$ & $2.04 \mathrm{ab}$ & $11.50 \mathrm{a}$ & $10.01 \mathrm{a}$ & $6.26 \mathrm{ab}$ \\
\hline & $\mathrm{T} 4$ & $18.40 \mathrm{~b}$ & $2.37 \mathrm{a}$ & $6.39 b$ & $38.30 \mathrm{ab}$ & $7.30 \mathrm{c}$ & $2.04 \mathrm{ab}$ & $12.60 \mathrm{a}$ & $9.97 \mathrm{a}$ & $6.46 \mathrm{a}$ \\
\hline & T5. & $19.80 \mathrm{a}$ & $2.10 \mathrm{a}$ & $6.98 \mathrm{a}$ & $42.70 \mathrm{ab}$ & $7.80 \mathrm{bc}$ & $2.04 \mathrm{~b}$ & $10.50 \mathrm{a}$ & $9.21 \mathrm{~b}$ & $5.49 \mathrm{~cd}$ \\
\hline & Mean & 18.00 & 2.21 & 6.42 & 37.80 & 6.84 & 2.03 & 11.30 & 9.70 & 5.90 \\
\hline
\end{tabular}

1) T1, Manda enzyme $(\times 10,000)$; T2, Yangmyeongwon $(\times 8,000)$; T3, effective microorganisms $(\times 3,000)$; T4, Kelpak $(\times 3,000)$; T5, control.

${ }^{2)}$ Mean values $\pm S D$ from separate experiments conducted in triplicate are shown. Mean with different letters (a-d) within the same growth characteristics are significantly different at $p<0.05$ by Duncan's multiple range test. 
A

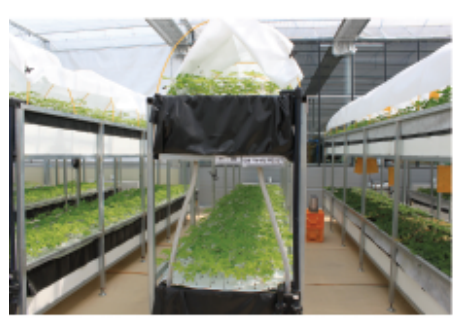

B

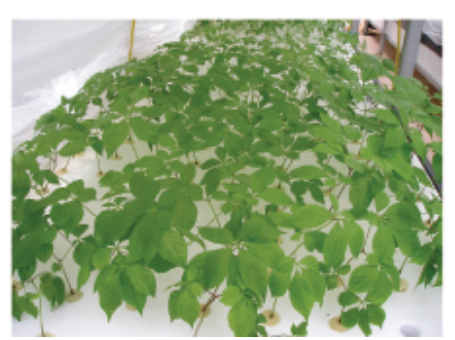

C

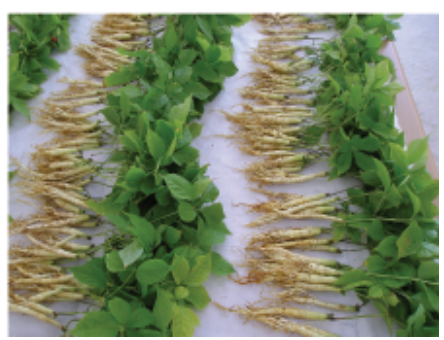

Fig. 1. Pictures of the aeroponic system using a two-layer vertical type of nutrient bath under natural light condition for ginseng culture (A,B) and harvested ginseng plants $(C)$. The total growth period was $120 \mathrm{~d}$. Ginseng plants are shown before harvesting (B) and after harvesting (C) on August 20, 2009.

A

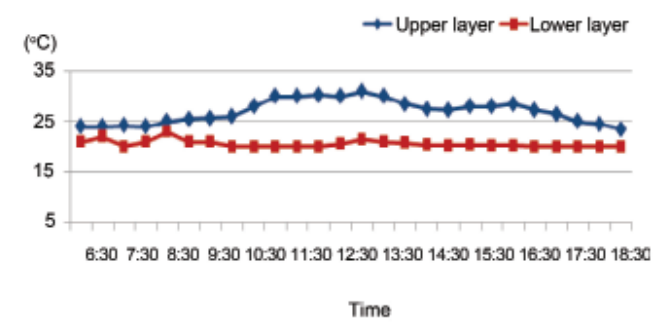

C

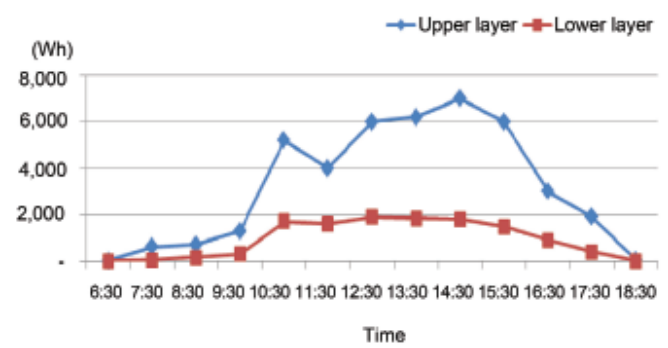

B

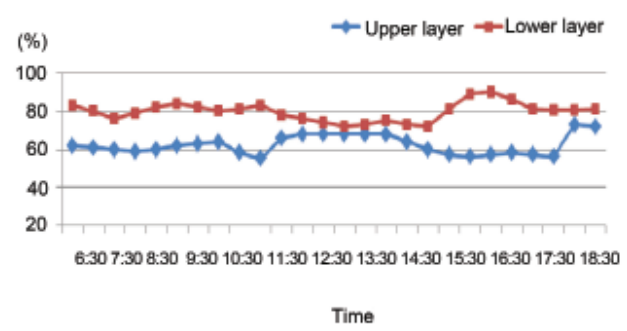

D

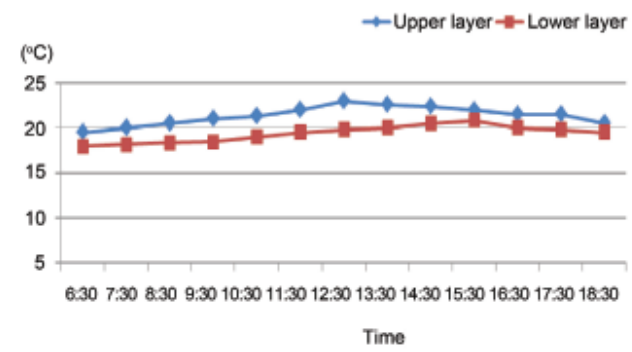

Fig. 2. Changes in the weather conditions such as temperature (A), humidity (B), solar radiation (C) and root zone temperature (D) in the daytime in the lower and upper layers of the greenhouse.

decreases in stem height, leaf length and chlorophyll content are more severe in one-year-old ginseng than 2-, 3-, or 4-year-old ginseng [16], light transmissibility should be one of the important environmental factors that influence the growth of ginseng in the aeroponic system with a short period of growing stages for one-year-old ginseng seedlings.

Unlike the growth of the aerial part of the ginseng, root growth, such as root weight and root diameter, was better in the upper layer with light intensity of 12,000 to $15,000 \mathrm{~lx}$ than in the lower layer of the aeroponic system, which coincides with the findings of the report that the weight of individual ginseng roots increases with an increase of light intensity [18], suggesting that the optimum light transmissibility for ginseng root yield should be $18.5 \%$ of natural light. In all treatments, the average root weight of the ginseng grown in the upper layer of the aeroponic system was 5.9 g, i.e., $13 \%$ more than ginseng grown in the lower layer, which showed an average root weight of $5.2 \mathrm{~g}$. Especially, the weight of the ginseng roots increased significantly in T3 (6.26 g) and T4 (6.46 g) compared to the other treatments and the untreated control. The average root length of the ginseng plants grown in the lower layer was $11.6 \mathrm{~cm}$, showing a slight increase compared to those in the upper layer with an average root length of $11.3 \mathrm{~cm}$; however, the average root diameter was comparably larger for the plants grown in the upper layer $(9.7 \mathrm{~mm})$ than those in the lower layer $(9.2 \mathrm{~mm})$, indicating that root diameter may be one of the major parameters contributing to the increase in the roots' weight. The plant growth of the aerial part and root differed depending on the upper and lower baths in which the ginseng seedlings were planted, regardless of the treatments. Examination of the indoor 
environmental conditions during the aeroponic culture of the ginseng seedlings showed that the natural light intensity was higher in the upper layer than in the lower layer (Figs. 1 and 2). Also, the indoor temperature was $2^{\circ} \mathrm{C}$ to $3^{\circ} \mathrm{C}$ higher in the upper layer $\left(25^{\circ} \mathrm{C}\right.$ to $\left.28^{\circ} \mathrm{C}\right)$ than in the lower layer $\left(23^{\circ} \mathrm{C}\right.$ to $\left.26^{\circ} \mathrm{C}\right)$. This suggests that ginseng root growth should be influenced more by light intensity and temperature than by treatment with natural bioactive products. However, the growth of the aerial part of the ginseng increased more in the lower layer baths of the aeroponic system, where there was a relatively low light intensity, which is unfavorable for ginseng root growth. All of the results suggest that proper management of light intensity and temperature would promote the production of specific parts of a ginseng plant to be used for specific purposes.

\section{Effects of natural bioactive products on the total ginsenoside content in ginseng plants cultured in a two-layer aeroponic system}

The results of the analysis of the total ginsenoside content of the ginseng roots and leaves cultured in the two-layer aeroponic system are shown in Fig. 3. In the untreated control (T5), the total ginsenoside content was higher in the ginseng roots cultured in the lower layer baths $(1.24 \%)$ than those cultivated in the upper layer, which does not coincide with the report that the crude saponin content in ginseng roots increases with an increase of light intensity [19]. On the other hand, the total ginsenoside content was significantly higher in the ginseng leaves cultured in the upper layer baths (16.57\%) than in the lower layer ones $(13.62 \%)$. Also, in the treatments with natural bioactive products (T1-T4), the total ginsenoside content in the ginseng roots and leaves was found to be the opposite of each other depending on whether they were cultured in the lower or the upper layer baths; i.e., higher in the leaves, lower in the roots, and vice versa. The total ginsenoside content of the ginseng roots in $\mathrm{T} 1$ and $\mathrm{T} 2$ came to $1.10 \%$ in the upper layer and $1.17 \%$ in the lower layer, respectively, i.e., higher than in the other layer in the same treatment; however, the content in T3 and T4 ranged from $1.23 \%$ to $1.30 \%$, showing no significant difference between the lower and upper layer baths.

In ginseng leaves, the total ginsenoside content in $\mathrm{T} 1$ was similar in the lower (15.33\%) and upper (14.23\%) layer baths of the aeroponic system; however, the content in T2-T4 came to $14.32,15.61$, and $15.11 \%$ in the ginseng leaves cultured in the upper layer baths, i.e., significantly higher than those cultured in the lower layer baths (Fig. 3). This seems to be due to the differential influ-

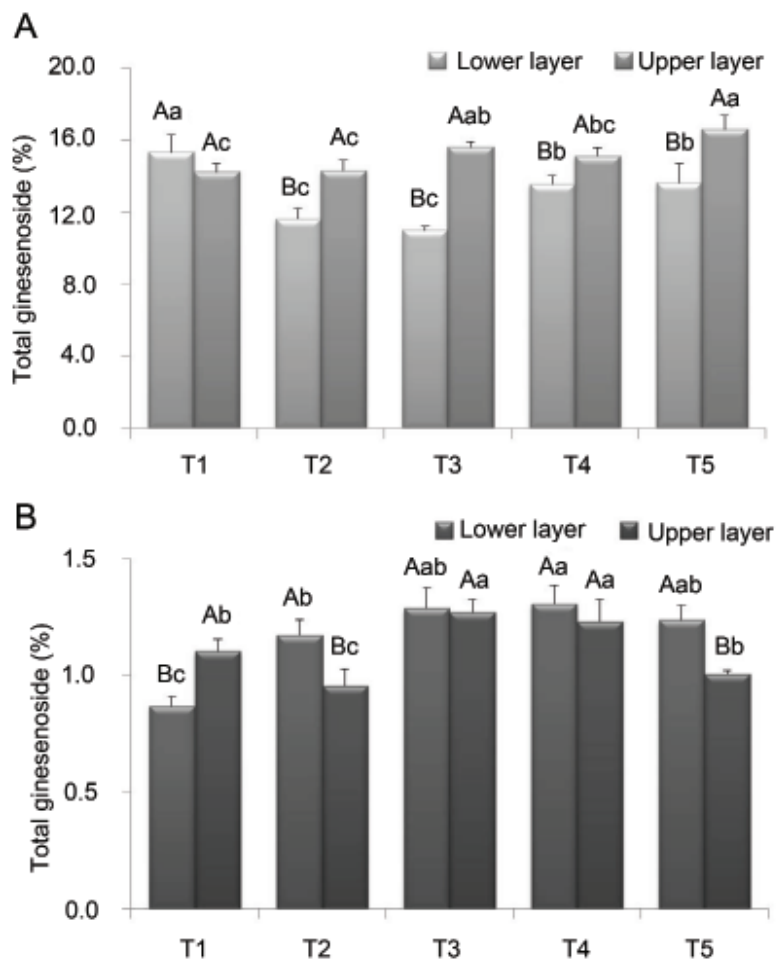

Fig. 3. The total ginsenoside content of the roots and leaves from Panax ginseng cultured using an aeroponic system with a two-layer vertical type of nutrient bath. (A) Ginsenoside content of leaves from $P$. ginseng cultured by hydroponics in the lower and upper layers of the greenhouse. (B) Ginsenoside content of roots from $P$. ginseng cultured by hydroponics in the lower and the upper layers of the greenhouse. T1-T5, natural bioactive products. T1, Manda enzyme $(\times 10,000)$; T2, Yangmyeongwon $(\times 8,000)$; T3, effective microorganisms $(\times 3,000)$; T4, Kelpak $(\times 3,000)$; T5, control. The mean values $\pm S D$ obtained from triplicate separate experiments are shown. Means with different letters $(A, B)$ within the same natural bioactive products are significantly different at $p<0.05$, while means with different letters $(\mathrm{a}-\mathrm{c})$ within the same layer are significantly different at $p<0.05$ by Duncan's multiple range test.

ences of the environmental conditions between the lower and upper layer baths. In particular, the total ginsenoside content of the leaves came to $15.61 \%$ and $16.57 \%$ in the upper layer baths treated with $\mathrm{T} 3$ and $\mathrm{T} 5$, respectively (with no significant difference between the two treatments), being significantly higher than in $\mathrm{T} 1$ and $\mathrm{T} 2$, but not between T3 and T4 (15.11\%). These results suggest that the treatments of T3 and T4 may be advantageous for the production of ginsenoside in the roots and leaves together in the upper-layer aeroponic system, while T1 may be advantageous for it in the lower-layer aeroponic system. On the other hand, the total ginsenoside content per unit weight of the ginseng roots and leaves in $\mathrm{T} 1$ was highest at $16.20 \%$ in the lower- layer baths of the aeroponic system among all the treatments, and was observed to decrease in the order of $\mathrm{T} 5, \mathrm{~T} 4, \mathrm{~T} 2$, and $\mathrm{T} 3$, at $14.86 \%$, 


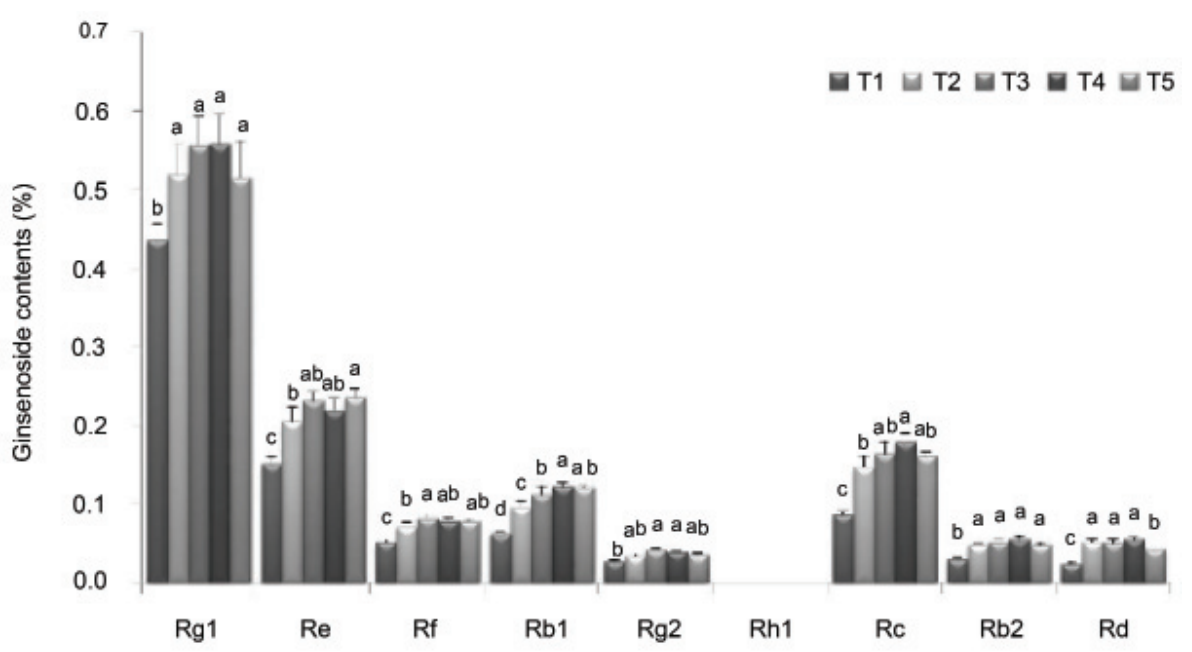

Fig. 4. Ginsenoside content of roots from Panax ginseng cultured in the lower layer of the aeroponic system using a two-layer vertical type of nutrient bath. T1-T5, natural bioactive products. T1, Manda enzyme $(\times 10,000)$; T2, Yangmyeongwon $(\times 8,000)$; T3, effective microorganisms $(\times 3,000)$; T4, Kelpak $(\times 3,000) ; \mathrm{T} 5$, control. Mean values \pm SD from triplicate separate experiments are shown. Means with different letters $(\mathrm{a}-\mathrm{c})$ within the same bioactive products are significantly different at $p<0.05$ by Duncan's multiple range test.

$14.84 \%, 13.21 \%$, and $12.30 \%$, respectively. Ginseng leaf growth is better in the lower than in the upper layer because the lower layer is advantageous for chlorophyll production and photosynthesis in ginseng leaves due to low light transmission [16,17]. In the lower layer culture conditions, plant photosynthesis might be enhanced by T1 (Manda enzyme), which contains 18 kinds of amino acids, minerals, and nutrients, consequently leading to accelerated plant growth presumably related to ginsenoside production. Manda enzyme, which is made from the 3-year-long fermentation of 50 kinds of fruits and vegetables, is a fermentation product with a variety of uses including agronomic and animal husbandry applications and as a health functional food. It was reported that it contains useful materials for humans owing to its antioxidant, antitumor and immune enhancement efficacies $[20,21]$. Its efficacies with regard to its immunostimulatory and growth stimulatory effects were also reported in the aquaculture of Japanese halibut [22]. On the other hand, the total ginsenoside content in ginseng plants cultured in the upper layer baths of the aeroponic system by the treatments (T1-T5) came to $15.33 \%, 15.28 \%$, $16.88 \%, 16.34 \%$, and $17.58 \%$, respectively, showing a decrease in ginsenoside production in the order of T5, $\mathrm{T} 3, \mathrm{~T} 4, \mathrm{~T} 1$, and $\mathrm{T} 2$. This suggests that natural bioactive products have different effects on ginsenoside production in ginseng roots and leaves depending on the upper and lower locations of the aeroponic system, or that the influence of T1 may not be very high in cultural conditions including high light transmission and high temperature.

\section{Production of ginsenoside components in ginseng plants cultured in the upper and lower layer baths of the aeroponic system and treated with natural bioactive products}

Fig. 4 shows the contents of ginsenoside components in ginseng roots cultured in the lower layer baths of the aeroponic system; the contents of the major ginsenoside components on average in T1-T5 were $\mathrm{Rg} 1>\mathrm{Re}>\mathrm{Rc}>\mathrm{Rb} 1>\mathrm{Rf}>\mathrm{Rb} 2>\mathrm{Rd}>\mathrm{Rg} 2$. The pattern of ginsenoside composition in the ginseng roots in the upper layer baths was the same as that in the lower layer baths of the aeroponic system (Fig. 5). However, the ginsenoside composition of the ginseng roots used in this study differs slightly from that produced in a hydroponic culture system with different nutrient media, in which the contents of the ginsenoside components were $\mathrm{Rg} 1>\mathrm{Rc}>\mathrm{Rb} 1>\mathrm{Re}>\mathrm{Rb} 2>\mathrm{Rf}>\mathrm{Rd}>\mathrm{Rh} 1$ [14]. The fact that the ginsenoside composition differs between aeroponic and hydroponic systems even in the untreated control suggests that the production level of individual ginsenoside components may differ depending on the system of cultivation. Given that the ginsenoside composition of 4-year-old ginseng roots cultivated in open fields is similar to those cultivated in a hydroponic system [15], ginseng cultivation in an aeroponic system may have a different influence on ginseng's efficacies compared to those grown in hydroponic or open field systems, altering the ginsenoside composition and especially decreasing the $\mathrm{PD} / \mathrm{PT}$ ratios due to the increased content of ginsenoside Re.

It is widely known that the expression of ginseng 


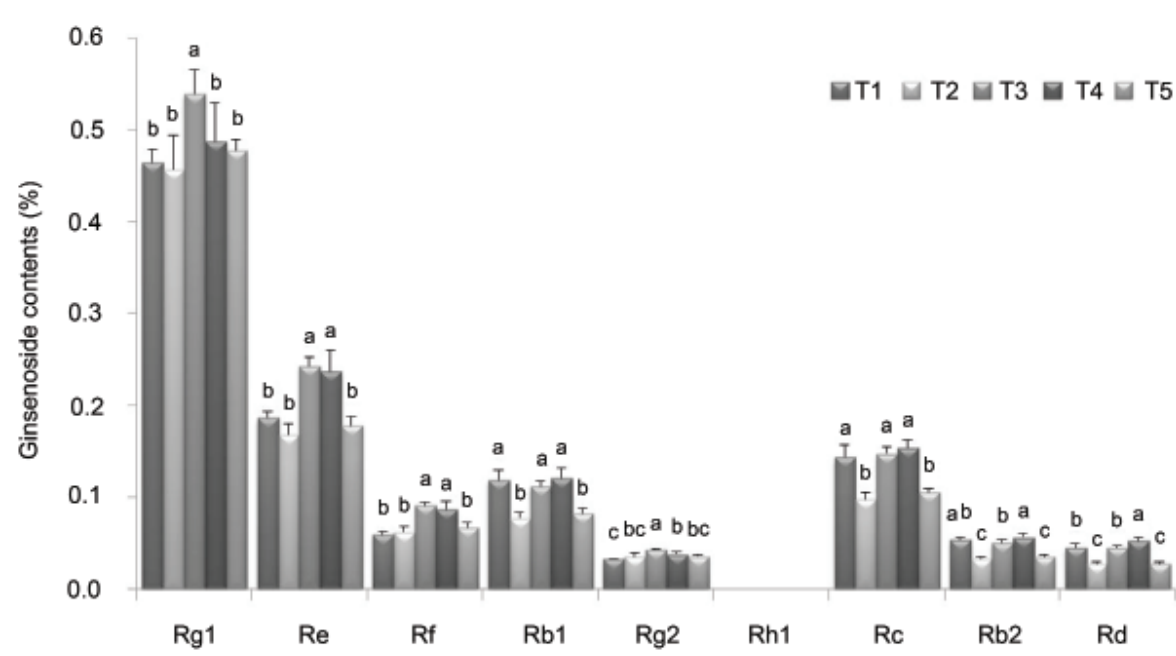

Fig. 5. Ginsenoside content of roots from Panax ginseng cultured in the upper layer of the aeroponic system using a two-layer vertical type of nutrient bath. T1-T5, natural bioactive products. T1, Manda enzyme $(\times 10,000)$; T2, Yangmyeongwon $(\times 8,000)$; T3, effective microorganisms $(\times 3,000)$; T4, Kelpak $(\times 3,000)$; T5, control. Mean values \pm SD from triplicate separate experiments are shown. Means with different letters (a-c) within the same bioactive products are significantly different at $p<0.05$ by Duncan's multiple range test.

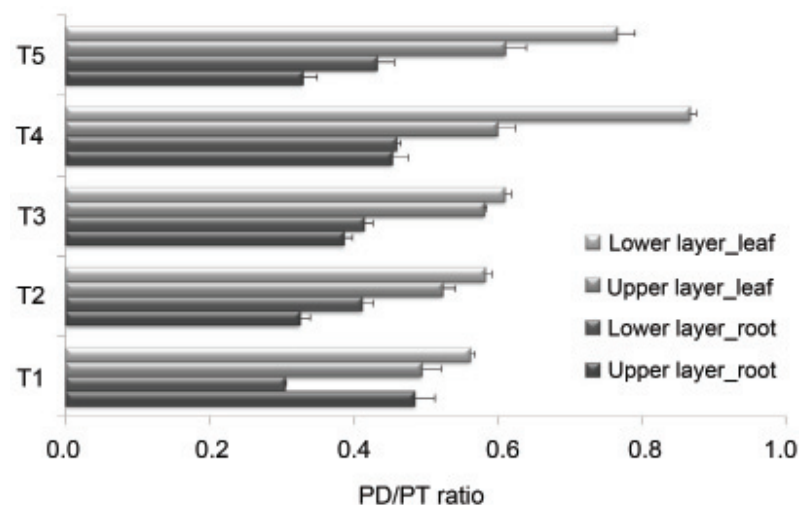

Fig. 6. Protopanaxadiol (PD)/ protopanaxatriol (PT) ratio of Panax ginseng cultured with the aeroponic system using a two-layer vertical type of nutrient bath. $P$. ginseng was cultured by the aeroponic system in the lower and the upper layers in a two-layer type of nutrient bath. PD/PT means the ratio of PD ginsenosides and PT ginsenosides. PD: Rb1, Rb2, Rc, Rd; PT: Re, Rf, Rg2, Rh1. T1-T5, natural bioactive products. T1, Manda enzyme $(\times 10,000)$; T2, Yangmyeongwon $(\times 8,000)$; T3, effective microorganisms $(\times 3,000)$; T4, Kelpak $(\times 3,000)$; T5, control. The mean values \pm SD obtained from triplicate separate-experiments are shown.

efficacies is especially governed not only by the total ginsenoside content but also by the composition of each individual ginsenoside components. Fig. 6 shows the protopanaxadiol (PD)/protopanaxatriol (PT) ratios in ginseng roots cultured in the aeroponic system and treated with natural bioactive products. In the untreated control, the ginsenoside $\mathrm{PD} / \mathrm{PT}$ ratios in the lower and upper layer baths were 0.44 and 0.33 , respectively, suggesting that the ginseng roots cultured in the lower layer baths with low light transmission contain more PD-type ginsenoside components than those cultured in the upper layer with high light transmission (in the aeroponic system). This is contrary to the report that the PD/PT ratios increase with an increase of light transmission [19], therefore suggesting that the $\mathrm{PD} / \mathrm{PT}$ ratios of the ginsenoside components may be influenced not only by light intensity but also by other minor cultural conditions. A further study is needed to understand the detailed relationships between ginsenoside production and composition with cultural conditions such as temperature and light transmission in the aeroponic system. On the other hand, the ginsenoside $\mathrm{Rc}$ and $\mathrm{Rb} 1$ contents were found to be higher in ginseng samples taken from the lower layer culture than the upper layer culture in the aeroponic system, with the former showing a higher percentage of ginsenoside Rc (13.0\%) among the total ginsenoside contents compared to a relatively low percentage $(10.4 \%)$ in those cultured in the latter.

The $\mathrm{PD} / \mathrm{PT}$ ratios of the ginseng roots treated with T2-T4 were higher in the lower layer baths than in the upper ones, showing a similar tendency to the untreated control. However, the PD/PT ratios in the ginseng roots treated with $\mathrm{T} 1$ were 0.31 and 0.49 for the lower and upper layer baths, respectively, showing higher $\mathrm{PD} / \mathrm{PT}$ ratios in the upper layer than in the lower one, i.e., contrary to the T2-T4 and the untreated control. Moreover, for T1, the PD/PT ratios in the upper layer were highest among all the treatments in either the upper or lower layer baths. Unlike other treatments, the total ginsenoside content of ginseng plants treated with $\mathrm{T} 1$ was higher in the upper layer, as shown in Fig. 3, which contained much higher 


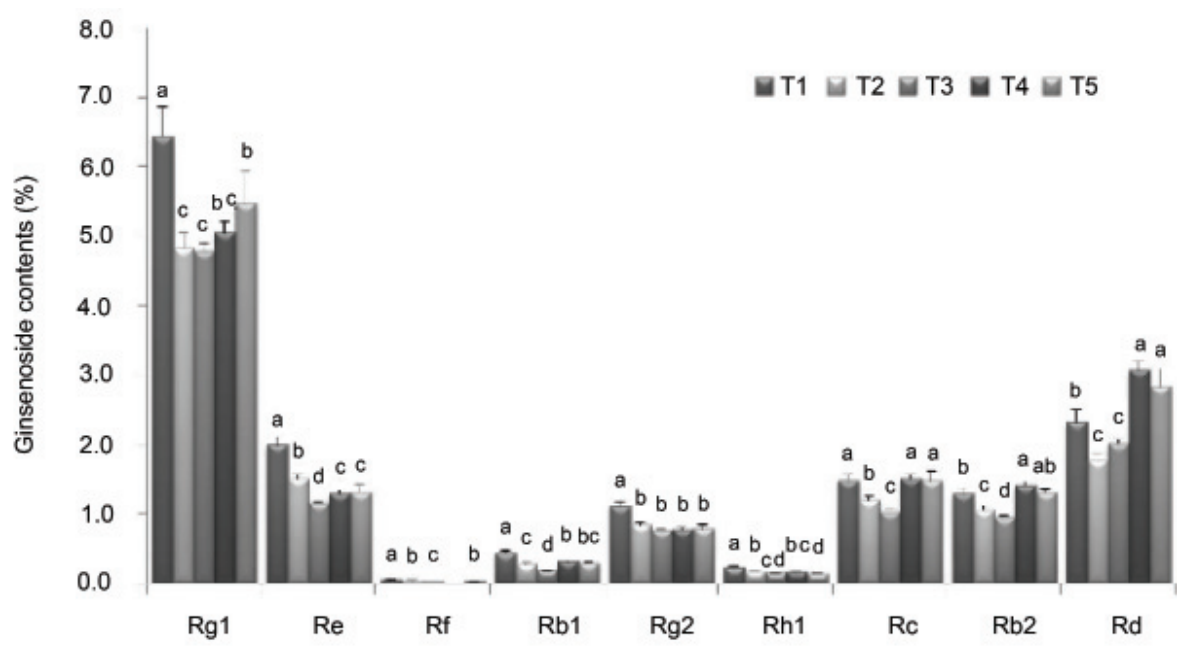

Fig. 7. Ginsenoside contents of leaves from Panax ginseng cultured in the lower layer of the aeroponic system using a two-layer vertical type of nutrient bath. T1-T5, natural bioactive products. T1, Manda enzyme $(\times 10,000)$; T2, Yangmyeongwon $(\times 8,000)$; T3, effective microorganisms $(\times 3,000)$; T4, Kelpak $(\times 3,000)$; T5, control. The mean values \pm SD obtained from triplicate separate experiments are shown. Means with different letters (a-c) within the same bioactive products are significantly different at $p<0.05$ by Duncan's multiple range test.

ginsenoside $\mathrm{Rb} 1$ and $\mathrm{Rc}$ contents than in the lower layer, accounting for $10.8 \%$ and $13.0 \%$ of the total ginsenoside content, respectively.

The PD/PT ratios in the hydroponically grown ginseng plants were around 0.6, i.e., lower than those grown in open fields [14]. In our study examining the effects of natural bioactive products on ginsenoside production, $\mathrm{T} 1$ induced greater production of ginsenoside $\mathrm{Rb} 1$ and $\mathrm{Rc}$ than the other treatments, thus increasing the $\mathrm{PD} /$ PT ratios. Ginsenoside Rb1 and Rc are known to have immunostimulatory effects and antianxiety efficacies $[12,23,24]$. Further detailed studies will be needed to examine the influence of $\mathrm{T} 1$ on ginsenoside production and composition in the upper and lower locations of an aeroponic system with different levels of light transmission.

Investigation of the individual ginsenoside components produced in the ginseng plants cultured in the lower layer of the aeroponic system showed the content of ginsenoside Rg1, the most significant ginsenoside component of a ginseng root, to be $0.52 \%$ to $0.56 \%$ in $\mathrm{T} 2-\mathrm{T} 5$, except in T1, which contained $0.44 \%$. T3 and T4, which similarly had the most prominent effect on the production of ginsenoside, had a similar effect, showing no significant difference in terms of the production of the 8 individual compeonents of ginsenoside. In the upper-layer aeroponic culture of the ginseng plants, there was no significant difference between $\mathrm{T} 3$ and $\mathrm{T} 4$ in terms of the total ginsenoside content; however, T3 contained $0.54 \%$ and $0.04 \%$ of ginsenoside $\mathrm{Rg} 1$ and $\mathrm{Rg} 2$, respectively, while $\mathrm{T} 4$ contained $0.06 \%$ and $0.05 \%$ of ginsenoside $\mathrm{Rb} 2$ and Rd, respectively (Fig. 5). There were no significant dif- ferences in the amounts of the other ginsenoside components in the ginseng plants cultured by $\mathrm{T} 3$ and $\mathrm{T} 4$ in the upper layer of the aeroponic system.

As regards the ginseng plants cultured in the lower layer of the aeroponic system, the ginsenoside Rg1 content was lowest $(0.44 \%)$ in the ginseng roots in $\mathrm{T} 1$, but it was significantly higher in the ginseng leaves in $\mathrm{T} 1$ than in the other treatments (Fig. 7). The contents of the major ginsenosides components produced in the ginseng leaves cultured in the lower layer of the aeroponic system were observed in the order of $\operatorname{Rg} 1>\mathrm{Rd}>\mathrm{Re}>\mathrm{Rc}>\mathrm{Rb} 2>\mathrm{Rg} 2>$ $\mathrm{Rb} 1>\mathrm{Rh} 1>\mathrm{Rf}$ (Fig. 7). The ginsenoside composition of the ginseng leaves cultured in the upper layer of the aeroponic system showed the same pattern as those cultured in the lower layer in our study (Fig. 5), and also in the hydroponic culture system in a previous study [14], suggesting that the composition of ginsenoside may not be greatly influenced by the aquiculture type (i.e., spray and substrate cultures) or by treatments with natural bioactive products.

The $\mathrm{PD} / \mathrm{PT}$ ratios were found to be 0.57 to 0.87 and 0.50 to 0.61 in the ginseng leaves cultured in the lowerand upper-layer baths of the aeroponic system, respectively, showing that they were higher in the lower layer than in the upper layer regardless of the treatments (Fig. 6). This tendency is similar to that observed in the ginseng roots, which suggests that the biosynthesis of PDgroup ginsenosides in ginseng plants may be higher in the lower layer baths with relatively low light intensity rather than in the upper layer of the aeroponic system. Especially high PD/PT ratios were shown in T4 and 


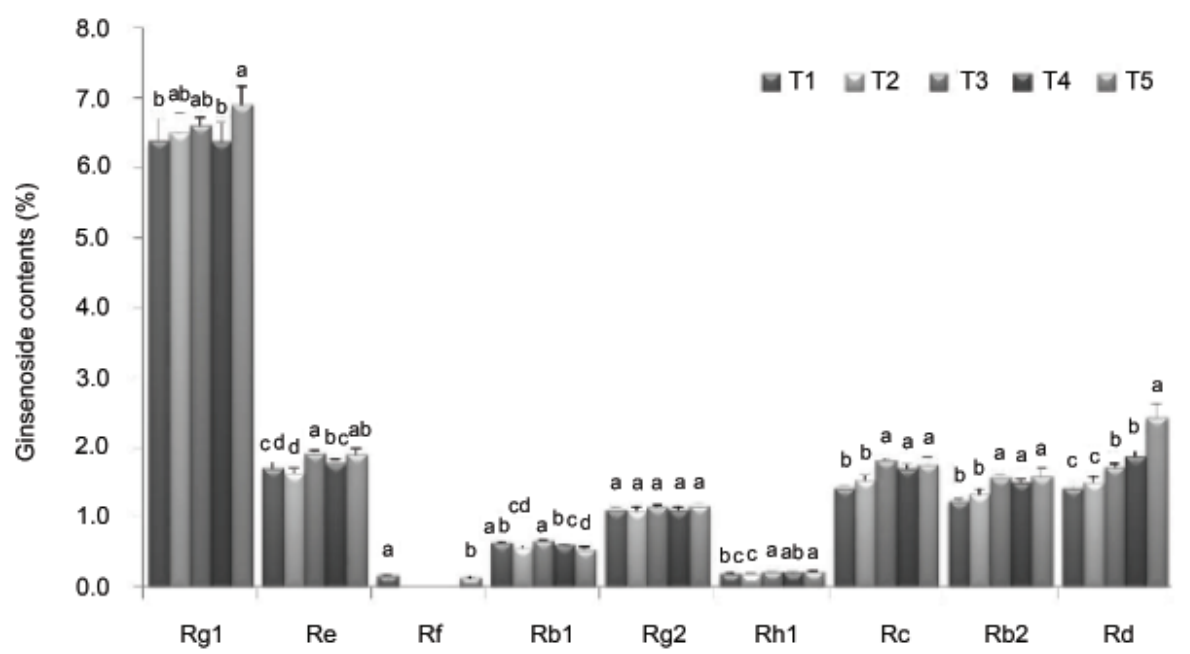

Fig. 8. Ginsenoside contents of leaves from Panax ginseng cultured in the upper layer of the aeroponic system using a two-layer vertical type of nutrient bath. T1-T5, natural bioactive products. T1, Manda enzyme $(\times 10,000)$; T2, Yangmyeongwon $(\times 8,000)$; T3, effective microorganisms $(\times 3,000)$; T4, Kelpak $(\times 3,000)$; T5, control. The mean values \pm SD obtained from triplicate separate experiments are shown. Means with different letters $(\mathrm{a}-\mathrm{c})$ within the same bioactive products are significantly different at $p<0.05$ by Duncan's multiple range test.

T5 (untreated control), indicating that their differences between the lower and upper layer baths were reduced in T1-T3, while they were increased in T4 compared to the untreated control (T5). This could be explained by the fact that the high PD-group ginsenoside Rd in the ginseng leaves $(3.07 \%)$ cultured in the lower layer baths was 1.6 times higher than those cultured in the upper layer (1.87\%) (Figs. 7 and 8 ). Ginsenoside Rd is a component known to be effective in enhancing antioxidant enzyme activities [25], cancer prevention [26], and brain cell protection [27]. T4 (Kelpak) is a bioactive substance extracted from brown algae (E. maxima), which has efficacies of plant growth promotion by enhancing rooting, flowering and fruiting [28-30] with the help of the plant hormones such as cytokinins and auxins it contains [31]. It is also useful for the mass production of commercial seaweed [32]. In our study, treatment with Kelpak might promote the growth of ginseng plants, which may have a positive influence on the production of $\mathrm{PD}$-group ginsenosides, especially ginsenoside Rd.

Presently several natural bioactive products are used for growth promotion and pest control in open-field cultivation of ginseng. However, there are few cases of experiments being conducted on the use of these natural bioactive products in the hydroponics and aeroponics of ginseng. In this respect, this is the first report on such experimentation to have been conducted in detail. Few studies have been conducted on the effect of natural bioactive products on ginsenoside production in open-field ginseng cultivation. Therefore, this study may provide basic information that can profitably be used in the application of natural bioactive products in open-field cultivation of ginseng as well as in hydroponic and aeroponic systems for ginseng production.

\section{ACKNOWLEDGEMENTS}

This work was supported in part by a grant (PJ006499) provided by the Rural Development Administration, Republic of Korea for projects involving the development of agricultural farming techniques for practical usage from the Rural Development Administration, Republic of Korea.

\section{REFERENCES}

1. Cheng JT. Merit of ginseng in the improvement of insulin resistance. J Ginseng Res 2010;34:155-159.

2. Vuksan V, Sievenpipper J, Jovanovski E, J AL. Current clinical evidence for Korean red ginseng in management of diabetes and vascular disease: a Toronto's ginseng clinical testing program. J Ginseng Res 2010;34:264-273.

3. Cho WC, Chung WS, Lee SK, Leung AW, Cheng CH, Yue KK. Ginsenoside Re of Panax ginseng possesses significant antioxidant and antihyperlipidemic efficacies in streptozotocin-induced diabetic rats. Eur J Pharmacol 2006;550:173-179.

4. Keum YS, Park KK, Lee JM, Chun KS, Park JH, Lee SK, Kwon H, Surh YJ. Antioxidant and anti-tumor promoting activities of the methanol extract of heat-processed gin- 
seng. Cancer Lett 2000;150:41-48

5. Kim HS, Lee EH, Ko SR, Choi KJ, Park JH, Im DS. Effects of ginsenosides $\operatorname{Rg} 3$ and $\mathrm{Rh} 2$ on the proliferation of prostate cancer cells. Arch Pharm Res 2004;27:429-435.

6. Suh SO, Boo YJ, Park JM, Kim J. Prospective study for Korean red ginseng extract as an immune modulator following a curative surgery in patients with advanced colon cancer. J Ginseng Res 2007;31:54-59.

7. Surh YJ, Na HK, Lee JY, Keum YS. Molecular mechanisms underlying anti-tumor promoting activities of heatprocessed Panax ginseng C.A. Meyer. J Korean Med Sci 2001;16 Suppl:S38-S41.

8. Van Kampen J, Robertson H, Hagg T, Drobitch R. Neuroprotective actions of the ginseng extract G115 in two rodent models of Parkinson's disease. Exp Neurol 2003; 184:521-529.

9. Lopez MV, Cuadrado MP, Ruiz-Poveda OM, Del Fresno AM, Accame ME. Neuroprotective effect of individual ginsenosides on astrocytes primary culture. Biochim Biophys Acta 2007;1770:1308-1316.

10. Kang KS, Yamabe N, Kim HY, Yokozawa T. Effect of sun ginseng methanol extract on lipopolysaccharide-induced liver injury in rats. Phytomedicine 2007;14:840-845.

11. Kwon YS, Jang KH. The effect of Korean red ginseng on liver regeneration after $70 \%$ hepatectomy in rats. J Vet Med Sci 2004;66:193-195.

12. Kim DH, Moon YS, Jung JS, Min SK, Son BK, Suh HW, Song DK. Effects of ginseng saponin administered intraperitoneally on the hypothalamo-pituitary-adrenal axis in mice. Neurosci Lett 2003;343:62-66.

13. Kim DH, Moon YS, Lee TH, Jung JS, Suh HW, Song DK. The inhibitory effect of ginseng saponins on the stress-induced plasma interleukin-6 level in mice. Neurosci Lett 2003;353:13-16.

14. Kim GS, Hyun DY, Kim YO, Lee SE, Kwon H, Cha SW, Park CB, Kim YB. Investigation of ginsenosides in different parts of Panax ginseng cultured by hydroponics. Korean J Hort Sci Technol 2010;28:216-226.

15. Kim GS, Hyun DY, Kim YO, Lee SW, Kim YC, Lee SE, Son YD, Lee MJ, Park CB, Park HK et al. Extraction and preprocessing methods for ginsenosides analysis of Panax ginseng C.A. Mayer. Korean J Med Crop Sci 2008; 16:446-454.

16. Lee JC, Cheon SK, Kim YT, Kim SD, Ahn SB. Studies on the optimum light intensity for growth of Panax ginseng. II. Study on the difference of the optimum light intensity for the growth of ginseng plant according to the root age. Korean J Ginseng Sci 1982;6:149-153.

17. Cheon SG, Lee TS, Yun JH, Lee SS. Effect of light transmittance control on the growth status of aerial parts dur- ing the growing season of Panax ginseng. J Ginseng Res 2003;27:202-206.

18. Lee CH, Lee JC, Cheon SK, Kim YT, Ahn SB. Studies on the optimum light intensity for growth of Panax ginseng. I. Effects of light intensity on growth of shoots and roots of ginseng plants. Korean J Ginseng Sci 1982;6:38-45.

19. Cheon SK, Mok SK, Lee SS. Effect of light intensity and quality on the growth and quality of Korean ginseng (Panax ginseng C. A. Meyer). III. Effects of light intensity on the quality of ginseng plant. Korean J Ginseng Sci 1991;15:144-151.

20. Hwang WI, Hwang YK, Lee JY, Lee JY, Okuda H. Antitumor and immunopotentiating effects of Manda enzyme. Nat Prod Sci 1996; 2:29-36.

21. Kim DC, Hwang WI, In MJ. In vitro antioxidant and anticancer activities of extracts from a fermented food. $\mathrm{J}$ Food Biochem 2003;27:449-459.

22. Ashima T, Okimasu E. Immunostimulatory effects of fermented vegetable product on the non-specific immunity of Japanese flounder Paralichthys olivaceus. Fish Sci 2005;71:257-262.

23. Luo YM, Cheng XJ, Yuan WX. Effects of ginseng root saponins and ginsenoside Rb1 on immunity in cold water swim stress mice and rats. Zhongguo Yao Li Xue Bao 1993;14:401-404.

24. Song X, Zang L, Hu S. Amplified immune response by ginsenoside-based nanoparticles (ginsomes). Vaccine 2009;27:2306-2311.

25. Yokozawa T, Liu ZW, Dong E. A study of ginsenoside$\mathrm{Rd}$ in a renal ischemia-reperfusion model. Nephron 1998;78:201-206.

26. Chang TL, Ding HY, Kao YW. Role of ginsenoside Rd in inhibiting 26S proteasome activity. J Agric Food Chem 2008;56:12011-12015.

27. Ye R, Li N, Han J, Kong X, Cao R, Rao Z, Zhao G. Neuroprotective effects of ginsenoside $\mathrm{Rd}$ against oxygenglucose deprivation in cultured hippocampal neurons. Neurosci Res 2009;64:306-310.

28. Crouch IJ, van Staden J. Effect of seaweed concentrate from Ecklonia maxima (Osbeck) Papenfuss on Meloidogyne incognita infestation on tomato. J Appl Phycol 1993;5:37-43.

29. Crouch IJ, Van Staden J. Commercial seaweed products as biostimulants in horticulture. J Home Consum Hortic 1993;1:19-76.

30. Kowalski B, Jager AK, Van Staden J. The effect of a seaweed concentrate on the in vitro growth and acclimatization of potato plantlets. Potato Res 1999;42:131-139.

31. Featonby-Smith BC, Van Staden J. Identification and seasonal variation of endogenous cytokinins in Ecklonia maxima (Os- 
Kim et al. Effects of Natural Bioactive Products on Panax ginseng in Aeroponic System

beck) Papenf. Bot Mar 1984;27:527-531.

32. Robertson-Andersson DV, Leitao D, Bolton JJ, Anderson RJ, Njobeni A, Ruck K. Can kelp extract (KEL-
PAK) be useful in seaweed mariculture? J Appl Phycol 2006;18:315-321. 